\title{
ANÁLISES DE AMOSTRAS AMBIENTAIS PARA FINS DE SALVAGUARDAS NUCLEARES NO BRASIL
}

\author{
Carina C. Lisbôa ${ }^{a, *,(1)}$, Olívio P. de Oliveira Júnior ${ }^{\mathrm{a}}$ e Delvonei A. de Andrade ${ }^{\mathrm{a}}$ \\ anstituto de Pesquisas Energéticas e Nucleares, Av. Prof. Lineu Prestes, 2242, 05508-000 São Paulo - SP, Brasil
}

Recebido em 29/06/2021; aceito em 05/10/2021; publicado na web em 12/11/2021

\begin{abstract}
ANALYSIS OF ENVIRONMENTAL SAMPLES FOR NUCLEAR SAFEGUARDS PURPOSES IN BRAZIL. The International Atomic Energy Agency (IAEA) is responsible for preventing the proliferation of nuclear weapons by detecting early the misuse of nuclear material or technology, and by certifying that States are honouring their safeguards obligations. The analysis of environmental samples taken by IAEA inspectors during inspections is a powerful tool for nuclear safeguards. This tool was implemented in 1996, after a successful application in Iraq, when a clandestine nuclear weapons program was discovered. The science involved in this type of analysis depends on highly sensitive and selective analytical measurements for detecting traces of nuclear materials collected in the nuclear facility's "environment". The purposes of this article are to draw attention to the relevance of this subject in order to address a significant global problem and to present what have been developed in Brazil. Through compatible results with certified standards and IAEA requirements, Brazil has already demonstrated the potential of measuring the amount and isotopic composition of uranium and plutonium at the levels expected in typical environmental samples. Finally, this work suggests the necessary measurements to implement a reliable and consistent environmental samples program for nuclear safeguards in Brazil.
\end{abstract}

Keywords: environmental samples; nuclear safeguards; plutonium; uranium.

\section{INTRODUÇÃO}

\section{A Agência Internacional de Energia Atômica e as salvaguardas nucleares}

A Agência Internacional de Energia Atômica (AIEA) é uma organização intergovernamental independente ligada à Organização das Nações Unidas (ONU) com sede em Viena, Áustria. ${ }^{1}$ Essa Agência foi criada em 1957 em resposta ao profundo temor mundial decorrente do domínio da tecnologia nuclear para fabricação de bombas com grande poder de destruição em massa. ${ }^{2}$

De acordo com seu Estatuto, os seus objetivos são de contribuir para acelerar e ampliar a contribuição da energia nuclear para a paz, saúde e prosperidade em todo o mundo e impedir a proliferação de armas nucleares..$^{3-5}$

As salvaguardas nucleares são medidas técnicas aplicadas pela AIEA com o objetivo de fornecer garantias confiáveis à comunidade internacional de que os materiais nucleares e outros itens especificados não são desviados do uso nuclear pacífico para fabricação de armas nucleares ou outros dispositivos nucleares explosivos..$^{1-4}$

As atividades de salvaguardas conduzidas pela AIEA são aplicadas em qualquer planta ou instalação do ciclo do combustível nuclear. Em geral, tais atividades incluem, entre outras:

- Inspeções físicas no local, podendo ser do tipo programada ou não programada;

- Contabilidade de materiais nucleares;

- Medições de material nuclear;

- Medidas de vigilância, contenção e monitoramento;

- Coleta e análise de amostras ambientais e de materiais nucleares; e

- Verificação do projeto da instalação. ${ }^{5}$

A base legal para atuação da AIEA provém de acordos internacionais assinados entre os Estados membros e a própria Agência. ${ }^{6}$ Tais acordos exigem compromissos vinculativos por parte dos Estados membros e estabelecem regimes apropriados de verificação de conformidade por parte da Agência. ${ }^{7}$

\section{Salvaguardas nucleares no Brasil}

A Comissão Nacional de Energia Nuclear (CNEN) foi criada em 1956 como uma autarquia federal para desenvolver a política nacional de energia nuclear com atribuições legais para regular, licenciar e fiscalizar a produção e o uso da tecnologia nuclear no Brasil. ${ }^{8}$

O Brasil se tornou um Estado membro da AIEA em 1957. ${ }^{6}$ Logo na década de 60 , já firmou os primeiros acordos de salvaguardas com a AIEA. ${ }^{9}$

Em 1991 foi criada a Agência Brasileiro-Argentina de Contabilidade e Controle de Materiais Nucleares (ABACC) e através do Acordo Quadripartite (INFCIRC/435), estabelecido entre o Brasil, a Argentina, a ABACC e a AIEA foi acordado que ambos os países prestarão apoio técnico à $\mathrm{ABACC}$ no cumprimento de seu papel de organismo regional de verificação de salvaguardas. Nesse contexto, a principal missão da ABACC é garantir à Argentina, ao Brasil e à comunidade internacional que todos os materiais e instalações nucleares existentes nos dois países estão sendo usados com fins exclusivamente pacíficos através da transparência aos seus programas nucleares, criando assim um ambiente de confiança mútua. ${ }^{10}$

Uma das mais recentes ações do Brasil com relação a salvaguardas nucleares foi a criação da Autoridade Nacional de Segurança Nuclear (ANSN) em maio de 2021. Essa concepção permitiu o desdobramento das competências da CNEN entre os dois órgãos: a ANSN ficará responsável pela regulação, fiscalização e licenciamento, e a CNEN conduzirá os trabalhos de execução de tarefas e pesquisas relacionadas ao uso da tecnologia nuclear. Tal ação foi de extrema importância para a comunidade internacional, pois de acordo com as normas internacionais, não é permitido que um mesmo órgão execute e também fiscalize as suas próprias atividades, como ocorria anteriormente. ${ }^{11}$

*e-mail: carinacerri@usp.br 


\section{Amostras ambientais como ferramenta de salvaguardas} nucleares

Desde a descoberta do programa clandestino de armas nucleares do Iraque em 1991, o sistema de salvaguardas da AIEA foi consideravelmente modificado, especialmente para fortalecer sua capacidade de detectar atividades e materiais nucleares não declarados. ${ }^{7}$

Antes de 1991, a comunidade internacional como um todo estava convencida de que os Estados que haviam assinado o Tratado de Não Proliferação (TNP) permaneceriam comprometidos com seus compromissos e, portanto, decidiram que o papel da Agência deveria se restringir à verificação de materiais e instalações declarados. Nesse sentido, nenhuma medida era executada de modo a detectar se um Estado estava tentando enganar o sistema através da realização de atividades não declaradas. ${ }^{7}$

Para abordar essa questão, a AIEA criou o "Programa 93 + 2" em 1993 com o objetivo de fortalecer o sistema de salvaguardas, tornando-o mais eficiente e eficaz e fornecendo métodos para detectar atividades e materiais nucleares clandestinos. Uma das principais medidas de fortalecimento estudadas no âmbito desse Programa foi a coleta e análise de amostras ambientais para detectar assinaturas nucleares que pudessem revelar atividades não declaradas. ${ }^{6,7,12}$

A AIEA passou então a aplicar rotineiramente a metodologia de coleta e análise de amostras ambientais em instalações nucleares a partir de 1996, tendo como base legal o âmbito de acordos de salvaguardas abrangentes e, de forma mais ampla, o Protocolo Adicional (INFCIRC/540 Corr.). ${ }^{7,13}$

A amostragem ambiental provou ser uma ferramenta poderosa e uma das mais fortes medidas de verificação técnica introduzidas para as salvaguardas da Agência. Enquanto muitas medidas de verificação de salvaguardas se esforçam para confirmar o tipo e a quantidade de material nuclear declarado por um Estado, essa ferramenta fornece um meio poderoso para detectar atividades e materiais nucleares não declarados e ajuda a AIEA a tirar conclusões confiáveis sobre as salvaguardas..$^{14}$

\section{A coleta e a análise de amostras ambientais}

A coleta de amostras ambientais é baseada no fato de que as instalações nucleares não são completamente vedadas e por isso liberam materiais particulados durante suas atividades e parte dessas partículas ficam no ambiente. ${ }^{15}$

Nesse cenário, uma amostra é retirada do meio ambiente a fim de analisá-la em busca de traços de compostos químicos, elementos ou isótopos que possam servir como um indicador relevante. Esse indicador pode ser idêntico ou parte de uma assinatura característica que é criada pela atividade ou instalação que está sendo investigada. ${ }^{7}$

As amostras são coletadas por inspetores treinados usando kits de amostragem padronizados e de acordo com os protocolos de amostragem específicos da instalação. ${ }^{16}$

Para a realização de coletas de amostras ambientais, os inspetores esfregam filtros de algodão, com dimensões e composição padronizadas, sobre superfícies localizadas em diversos pontos da instalação, e por isso, tais amostras também são denominadas de "esfregaços".

A distinção entre atividades nucleares declaradas e aquelas que podem ser clandestinas só pode ser constatada por meio de dados de medição de alta sensibilidade, qualidade e confiabilidade. ${ }^{14} \mathrm{~A}$ ciência envolvida na análise de amostras ambientais para salvaguardas depende de medições analíticas em instrumentos altamente sensíveis e seletivos para detecção de traços e vestígios de materiais nucleares coletados no "meio ambiente" de uma instalação nuclear. ${ }^{13}$
Por se tratar de dados de relevância internacional, os requisitos de precisão de medições realizadas na área de salvaguardas de materiais nucleares devem estar claramente definidos. No caso das medições em amostras ambientais, os requisitos estão apresentados na Tabela $1 .{ }^{17}$

Tabela 1. Requisitos de desempenho analítico de medições em amostras ambientais (10 ng de $\mathrm{U}$ e $0,1 \mathrm{pg}$ de $\mathrm{Pu})^{17}$

\begin{tabular}{cc}
\hline Grandeza Medida & Requisito de Precisão \\
\hline Quantidade de U & $\leq 10 \%$ \\
Quantidade de Pu & $\leq 10 \%$ \\
$\mathrm{U}-235$ / U-238 & $\leq 1 \%$ \\
$\mathrm{U}-234$ / U-238 & $\leq 10 \%$ \\
$\mathrm{U}-236 / \mathrm{U}-238$ & $\leq 10 \%$ \\
$\mathrm{Pu}-240 / \mathrm{Pu}-239$ & $\leq 10 \%$ \\
\hline
\end{tabular}

Atualmente tais requisitos podem ser alcançados pelas técnicas de Espectrometria de Massa com Plasma Indutivamente Acoplado (ICPMS) e Espectrometria de Massa por Ionização Térmica (TIMS). ${ }^{18}$

A espectrometria de massas é uma técnica analítica físico-química empregada para detectar e identificar átomos / moléculas de interesse com o objetivo de caracterizar a estrutura da matéria. Nesta análise, as moléculas em uma amostra são convertidas em íons em fase gasosa e o movimento desses íons através de um campo elétrico, magnético ou em ambos simultaneamente ocorre de modo a separá-los de acordo com a sua relação massa/carga $(\mathrm{m} / \mathrm{z})$.

\section{Informações extraídas a partir da análise das amostras de esfregaço}

Uma quantidade verdadeiramente surpreendente de informações pode ser obtida a partir da pequena quantidade de material coletado em uma amostra ambiental por esfregaço. A análise de tais amostras pode determinar "impressões digitais" ou "assinaturas únicas" de atividades nucleares, revelando informações e indicadores de atividades passadas e atuais em locais que manuseiam ou manusearam materiais nucleares. ${ }^{13}$

Para salvaguardas de materiais nucleares, os principais indicadores são a presença de urânio ou plutônio, pois possibilitam a associação do material com atividades nucleares, as quais estão vinculadas aos processos do ciclo do combustível. ${ }^{6,14}$

No caso do plutônio, trata-se de um elemento não existente de maneira natural, mas sim produzido pelo produto da reação de fissão do urânio. A reação nuclear de fissão do plutônio libera ainda mais energia do que a fissão do urânio, sendo, portanto, uma grande preocupação mundial pela possibilidade de emprego na fabricação de armamentos nucleares.

A presença de plutônio em análises de amostras ambientais pode ser proveniente do uso de combustível nuclear do tipo MOX (mistura de $\mathrm{UO}_{2}$ e $\mathrm{PuO}_{2}$ ), de combustível nuclear já irradiado ou de unidades de reprocessamento. ${ }^{6} \mathrm{O}$ reprocessamento é um processo constituído por operações químicas e físicas realizadas com o objetivo de separar urânio e plutônio no combustível irradiado, o que permite a sua reutilização no ciclo do combustível nuclear. ${ }^{15}$

No caso do urânio, existem três isótopos estáveis do elemento urânio de número atômico 92. Os isótopos naturais de urânio possuem número de massa 234, 235 e 238, cuja proporção em abundância atômica destes isótopos está apresentada na Tabela $2 .{ }^{19}$

No entanto, o urânio pode entrar no meio ambiente como consequência de diversas atividades humanas (processos antropogênicos), especialmente as que estão voltadas para o emprego da tecnologia nuclear. $^{20}$ 
Tabela 2. Composição isotópica do urânio natural ${ }^{19}$

\begin{tabular}{cc}
\hline Isótopo & $\%$ atômica \\
\hline U-234 & $0,0055 \%$ \\
U-235 & $0,7200 \%$ \\
U-238 & $99,27 \%$ \\
\hline
\end{tabular}

A descoberta de que, entre os isótopos de ocorrência natural, apenas o de massa 235 sofre uma reação nuclear em cadeia determinou a necessidade de obter urânio enriquecido artificialmente. Assim, a natureza das operações da instalação é parcialmente revelada na composição isotópica de urânio e, portanto, a determinação confiável desta composição passou a ser uma das medidas analíticas mais importantes do ciclo do combustível nuclear. ${ }^{19}$

O urânio também tem outros onze isótopos artificiais, dos quais aqueles de massa 233 e 236 são os mais importantes. ${ }^{19} \mathrm{O}$ U-233, que é produzido pela irradiação de nêutrons de Th-232, é um isótopo fissionável, assim como o U-235. Já, o U-236 é produzido na queima do reator, uma vez que é um isótopo produzido por capturas de nêutrons a partir da irradiação do U-235. ${ }^{6}$

A verificação do urânio nas amostras ambientais é normalmente baseada na razão U-235/U-238 por ser este o indicador de enriquecimento. As relações de abundância atômica desses isótopos dependem da extensão do processo de enriquecimento e podem ser usadas para distinguir as fontes naturais de urânio das fontes antropogênicas. ${ }^{12,20}$

Informações adicionais são obtidas a partir da presença de partículas de U-236. A quantidade do isótopo artificial U-236 pode ser um indicativo de que o material foi irradiado em um reator nuclear ou pode ser indício de uma atividade de reprocessamento. ${ }^{6}$

\section{Etapas do processo de amostragem ambiental}

A identificação dos pontos de amostragem em uma instalação é determinada no estágio de planejamento e depende dos objetivos das salvaguardas a serem atendidos, bem como do projeto específico da instalação e das informações operacionais. ${ }^{16}$

Os dados de medição da análise laboratorial das amostras são relatados ao Departamento de Salvaguardas da AIEA. Estes resultados são comparados com a assinatura nuclear esperada para a instalação amostrada, levando em consideração valores previstos para vários cenários envolvidos em atividades relacionadas ao ciclo do combustível nuclear. As assinaturas esperadas dessas atividades ou materiais declarados são determinadas e comparadas aos resultados analíticos passados e presentes do local amostrado. ${ }^{14,16}$

Quando os resultados estão de acordo com as declarações da instalação, eles fornecem garantia adicional de que nenhum uso indevido ocorreu. Quando uma possível discrepância ou inconsistência é encontrada, ações são tomadas para confirmá-la, por exemplo, verificando novamente os resultados pelo laboratório, analisando as amostras de arquivo em outro laboratório ou coletando novas amostras. $\mathrm{O}$ avaliador dos dados deve estar bem informado sobre as limitações dos métodos de análise e deve estar constantemente alerta para dados enganosos causados por possíveis contaminações, interferências ou erros. ${ }^{13,16}$

\section{Metodologias de preparação das amostras para realização das análises}

Como as amostras ambientais normalmente contêm apenas traços de materiais nucleares, requisitos rigorosos para preparação de amostras são necessários para obter resultados confiáveis. As técnicas analíticas de espectrometria de massas para análise de amostras ambientais requerem o emprego de amostras altamente purificadas, livres de interferências como orgânicos e metais pesados, para garantir a qualidade da medição de alta precisão. ${ }^{18,21}$

As amostras de esfregaços retiradas de instalações nucleares geralmente contêm algo em torno de nanogramas de urânio e/ou plutônio. Considerando esse baixo teor dos elementos de interesse presentes nas amostras, existe um grande potencial de contaminação durante o processamento químico de uma amostra em um ambiente típico de laboratório.

Nesse contexto, a recomendação é a de que o manuseio e a preparação das amostras sejam realizados em ambientes de laboratório com classificação de sala limpa. Se salas limpas não estiverem disponíveis, um rigoroso controle de limpeza do laboratório deve ser estabelecido e mantido. Nesse caso, o ideal é que a preparação de amostras seja realizada em um laboratório que não manipule outros tipos de amostras nucleares e que o manuseio das amostras seja executado em capelas de fluxo laminar que filtram o ar por meio de filtros HEPA (High Efficiency Particulate Air).

As amostras ambientais coletadas por esfregaço podem ser analisadas em sua totalidade (bulk analysis) ou em partículas individuais (particle analysis). ${ }^{15} \mathrm{~A}$ análise bulk envolve a digestão total do esfregaço e é realizada para medição da composição isotópica média para todo material contido na amostra. Por outro lado, a análise de partículas envolve a medição de partículas individuais de modo a obter informações mais específicas da partícula analisada. . 22,23

O método de preparação de amostras mais usual é o que faz a análise do esfregaço em sua totalidade, também chamado de processo de Digestão Total, já que o esfregaço é totalmente digerido em uma solução ácida. ${ }^{6}$

Nesse método, a determinação de urânio e plutônio em amostras ambientais por espectrometria de massas requer vários procedimentos para separar esses radionuclídeos da matriz. As amostras ambientais podem ser totalmente dissolvidas por digestão por micro-ondas ou lixiviadas em ácidos fortes. Em seguida, são realizadas metodologias químicas de separação e purificação das amostras. Essas metodologias normalmente são realizadas de modo manual e adicionam vários dias ao tempo de preparação das amostras para análise. Muitas técnicas para a separação são baseadas em cromatografia de troca iônica, extração por solvente ou cromatografia de extração. Especialmente, a aplicação da cromatografia de extração tem sido intensamente explorada devido à disponibilidade de resinas de extração TEVA ${ }^{\circledR} \mathrm{e}$ UTEVA $^{\circledR}$ da empresa Eichrom Industries Inc. ${ }^{13,18,20}$

Recentemente, pesquisadores do Oak Ridge National Laboratory (ORNL) desenvolverem um equipamento denominado de prepFAST em parceria com a empresa Elemental Scientific Inc. (ESI). Esse equipamento tem como objetivo automatizar e encurtar os procedimentos e métodos manuais e dispendiosos de preparação de amostras. Ele é basicamente constituído por um sistema de válvulas e bombas que controlam o fluxo da amostra ao longo do processo que contém colunas com as resinas TEVA ${ }^{\circledR}$ e UTEVA ${ }^{\circledR}$ para purificação e separação de urânio e plutônio nas amostras. ${ }^{18,21}$

\section{Os Laboratórios Analíticos da AIEA}

Os inspetores coletam várias centenas de amostras ambientais por ano em instalações nucleares. Estas amostras são analisadas no laboratório da AIEA (Environmental Sample Laboratory - ESL), localizado em Seibersdorf na Áustria, e em outros laboratórios localizados ao redor do mundo, pertencentes à Estados signatários dos acordos de salvaguardas, integrantes da Rede de Laboratórios Analíticos da AIEA (Network of Analytical Laboratories - NWAL). ${ }^{13}$ 
Diante da limitada capacidade de processamento de amostras por parte do Laboratório da AIEA, julgou-se interessante credenciar laboratórios ao redor do mundo. Nesse sentido, a AIEA procura ativamente uma participação mais ampla em sua rede de laboratórios que podem fornecer serviços analíticos de alta qualidade. A ampliação da rede serve para melhor distribuir a demanda de análise das amostras coletadas nas inspeções e, assim, reduzir os tempos de resposta. Ademais, a análise paralela de amostras replicadas em diferentes laboratórios é uma medida de garantia de qualidade e confiabilidade importante para mitigar possíveis erros laboratoriais. ${ }^{24}$

Nos últimos anos, a AIEA tem empreendido esforços num projeto de melhoria da capacitação de serviços analíticos para fins de salvaguardas. Esse projeto, denominado Enhancing Capabilities of the Safeguards Analytical Services (ECAS), engloba não só investimentos para aquisição de equipamentos e modernização dos seus laboratórios próprios e da rede de laboratórios credenciados, como também engloba a qualificação de outros laboratórios em Estados membros para realização de análises ambientais e análises de caracterização do material nuclear. ${ }^{3}$

\section{A capacitação do Brasil para realização de análises em amostras ambientais para fins de salvaguardas nucleares}

Embora tenham sido poucos os trabalhos realizados no Brasil em análises de amostras ambientais para fins de salvaguardas nucleares, estes trabalhos são de grande relevância por demonstrarem a capacitação brasileira para realização desse tipo de análise. A pequena quantidade de trabalhos publicados deve-se aos seguintes fatores: elevado custo da infraestrutura laboratorial e dos instrumentos analíticos, necessidade de profissionais especializados na análise de elementos a níveis de traços, baixa demanda de análises a serem executadas anualmente e, principalmente, ao sigilo que cerca este tipo de assunto, o que acaba sempre dificultando a publicação de resultados experimentais.

José M. Godoy do Instituto de Radioproteção e Dosimetria (IRD), CNEN, foi um dos primeiros pesquisadores brasileiros a realizar trabalhos com amostras ambientais para medições de radioisótopos de interesse. Na década de 80, o autor publicou um método analítico sequencial para separação desses radioisótopos. ${ }^{25}$ Já na década seguinte, o método foi aperfeiçoado. ${ }^{26}$

Com relação ao histórico de desenvolvimento da capacitação analítica brasileira para medição de amostras ambientais para fins de salvaguardas, é importante mencionar o programa de cooperação iniciado na década de 90 pela ABACC com a assistência do Departamento de Energia dos Estados Unidos (Department of Energy - DOE). O programa foi concebido para melhorar a competência técnica do Brasil e da Argentina na realização de análises de amostragem ambiental voltadas para atividades de salvaguardas, no qual foram realizados treinamentos de pessoal e exercícios de comparação interlaboratorial. ${ }^{17,27}$ Os laboratórios participantes desse programa estão relacionados na Tabela 3.

A primeira etapa do programa foi realizada para verificar a capacidade dos laboratórios em medir a quantidade e a composição isotópica do urânio em níveis esperados de serem encontrados em amostras ambientais típicas. De modo similar, foi realizada uma segunda etapa para medições da quantidade e da composição isotópica de plutônio. Por fim, foi realizada uma terceira etapa para medição de urânio e plutônio em uma mesma amostra, exigindo a necessidade de emprego de técnicas de separação e purificação desses elementos químicos. ${ }^{17,27}$

Godoy et al. publicaram trabalhos de medições das concentrações e das composições isotópicas de urânio e plutônio que estão inseridos nesse programa e demonstrou o excelente desempenho do IRD ao atingir os requisitos de precisão para medições em amostras ambientais apresentados na Tabela 1..$^{28,29}$

Já com relação a trabalhos acadêmicos, um dos trabalhos brasileiros pioneiros foi a tese de doutoramento de José H. Buchmann realizada no Instituto de Pesquisas Energéticas e Nucleares (IPEN) sobre o uso da técnica de bioindicadores para fins de salvaguardas. Nesse trabalho, o pesquisador utilizou agulhas de pinheiros coletadas em localidades próximas a uma instalação que manipula urânio situada dentro do Campus Universitário da Universidade de São Paulo. ${ }^{9}$

Outros dois trabalhos acadêmicos que merecem destaque na área de medições de amostras ambientais para fins de salvaguardas são as dissertações de Rafael C. B. Pestana e Rafael C. Marin, ambos realizados no IPEN. Nesses trabalhos foram coletadas amostras de esfregaços reais em instalações nucleares do IPEN e foram realizadas medições da composição isotópica do urânio por ICP-MS. ${ }^{6,15}$

\section{MATERIAIS E MÉTODOS}

No método analítico sequencial para separação de radioisótopos de interesse desenvolvido por Godoy et al., os isótopos de urânio e tório foram inicialmente separados usando óxido de tri-n-octil fosfina (TOPO) suportado em coluna de sílica gel. Os isótopos de urânio foram determinados por espectrometria alfa após outra extração com TOPO, porém realizada em uma membrana polimérica. Isótopos de tório foram co-precipitados com fluoreto de lantânio antes da medição em um espectrômetro alfa. Os isótopos de rádio foram separados por co-precipitação em uma solução de sulfato de chumbo e bário e determinados por técnicas de medição de radioatividade. ${ }^{25}$ Já no trabalho de melhoria do método publicado mais tarde por Godoy et al., foi depositado cobre para melhorar o

Tabela 3. Relação de laboratórios participantes do programa desenvolvido pela ABACC com assistência do DOE

\begin{tabular}{|c|c|c|}
\hline Laboratório & Instituição & Localização \\
\hline Instituto de Radioproteção e Dosimetria - IRD & Comissão Nacional de Energia Nuclear - CNEN & Rio de Janeiro, Brasil \\
\hline Laboratório de Análises Químicas & Dioxitek & Córdoba, Argentina \\
\hline Laboratório de Medições Ambientais & Autoridade Regulatória Nuclear - ARN & Buenos Aires, Argentina \\
\hline Laboratório de Caracterização Quimica & Instituto de Pesquisas Energéticas e Nucleares - IPEN & São Paulo, Brasil \\
\hline Departamento de Radioproteção Ambiental & Instituto de Pesquisas Energéticas e Nucleares - IPEN & São Paulo, Brasil \\
\hline Laboratório de Análises Químicas & Comissão Nacional de Energia Atômica - CNEA & Buenos Aires, Argentina \\
\hline Laboratório de Caracterização de $\mathrm{UF}_{6}$ & Centro Tecnológico da Marinha em São Paulo - CTMSP & São Paulo, Brasil \\
\hline Pacific Northwest National Laboratory - PNNL & Department of Energy - DOE & Richland - Washington / EUA \\
\hline Lawrence Livermore National Laboratory - LLNL & Department of Energy - DOE & Livermore - California / EUA \\
\hline Oak Ridge National Laboratory - ORNL & Department of Energy - DOE & Oak Ridge - Tennesse / EUA \\
\hline
\end{tabular}


fator de descontaminação de polônio no tório, além da inclusão de outras etapas que permitiram a identificação de chumbo na amostra. ${ }^{26}$

A primeira etapa do programa estabelecido entre a ABACC e o DOE foi dividida em três fases: 1) avaliação quantitativa do branco de urânio em cada estágio do processo de preparação e análise da amostra; 2) medição do urânio total e isotópico em folhas de pêssego que continham uma concentração de urânio conhecida; e 3) medição de amostras de esfregaço preparadas pela AIEA. ${ }^{27}$

No exercício de medição de amostras de esfregaço preparadas pela AIEA, cada laboratório recebeu cinco conjuntos de amostras preparados em triplicata (15 amostras no total), possuindo em cada conjunto: uma amostra para branco, duas amostras com aproximadamente $3 \%$ de U-235 e duas amostras com aproximadamente $20 \%$ de U-235. Para a medição das amostras, os laboratórios empregaram um ICP-MS. Apenas os laboratórios do DOE empregaram um ICP-MS e um TIMS com o propósito de comparação dos resultados. ${ }^{27}$

A segunda etapa do programa contou com a medição de materiais de referência de plutônio do New Brunswick Laboratory (NBL) e de amostras preparadas pelo Institute of Reference Materials and Measurements (IRMM). ${ }^{17}$

Dentro do programa da ABACC em parceria com o DOE em sua terceira fase, Godoy et al. desenvolveu uma metodologia analítica com um procedimento de separação de plutônio e urânio baseado em cromatografia de extração usando colunas TEVA ${ }^{\circledR}$ e UTEVA ${ }^{\circledR}$. As medições de concentração e composição isotópica foram realizadas em um ICP-MS aplicando um nebulizador ultrassônico acoplado a um sistema de dessolvatação por membrana. ${ }^{28,29}$

Nestes trabalhos, materiais de referência de plutônio e urânio certificados foram usados para testes de precisão e exatidão da razão atômica. Como amostras de teste, foram empregadas amostras preparadas pelo Lawrence Livermore National Laboratory (LLNL) e foram realizadas comparações entre os resultados desses dois laboratórios (IRD e LLNL). ${ }^{28,29}$

No trabalho de Buchmann, as medições das amostras de agulha de pinheiro foram preparadas por três métodos distintos de processamento: 1) lavagem ácida assistida por ultrassom; 2) digestão ácida assistida por micro-ondas; e 3) lixiviação ácida. Em todos esses métodos de processamento, a matriz foi dissolvida em uma solução de ácido nítrico ultrapuro e foram obtidas soluções capazes de serem introduzidas no instrumento de análise, um ICP-MS de alta resolução (HR-ICP-MS). ${ }^{9}$
Nos trabalhos de Pestana e Marin foram realizadas medidas de atividade $\alpha$, caracterização morfológica por Microscopia Eletrônica de Varredura com Sistema de Energia Dispersiva (MEV-EDS) e caracterização isotópica por ICP-MS. No entanto, há uma diferença considerável entre estes trabalhos com relação à metodologia de preparação das amostras introduzidas no instrumento de análise isotópica. ${ }^{6,15}$

Pestana utilizou a técnica na qual os esfregaços são digeridos em sua totalidade (bulk analysis) e, portanto, a medição da composição isotópica corresponde à média de todo material contido na amostra. $\mathrm{O}$ autor empregou duas metodologias distintas de preparação das amostras: lixiviação assistida por ultrassom e digestão total. Cada esfregaço coletado foi dividido ao meio e cada metade foi preparada por uma das metodologias. ${ }^{6}$

Por outro lado, Marin realizou a técnica de medição de partículas individuais (particle analysis) através do emprego de um sistema de ablação a laser acoplado ao ICP-MS (LA-ICP-MS). ${ }^{15}$

Esses dois últimos autores publicaram um trabalho em conjunto de comparação entre as técnicas ICP-MS e LA-ICP-MS para análise de urânio em amostras reais de esfregaço. As amostras foram coletadas em réplica em três pontos em uma instalação do IPEN e cada amostra foi analisada por uma das técnicas mencionadas. ${ }^{30}$

A Tabela 4 resume as principais informações dos trabalhos citados.

\section{RESULTADOS E DISCUSSÃO}

Através do trabalho realizado por Godoy et al., de desenvolvimento de um método analítico sequencial para separação de radioisótopos de interesse, foi possível realizar medições desses isótopos em amostras ambientais. ${ }^{25,26}$

Os resultados isotópicos obtidos na primeira etapa do programa estabelecido entre a ABACC e o DOE apresentaram concordância para os isótopos U-235 e U-238. Alguns laboratórios obtiveram problemas de fundo ou falta de sensibilidade para os resultados dos isótopos de urânio de baixas concentrações: U-234 e U-236. Para as amostras com maiores quantidades de urânio, esse problema se tornou menos perceptível. ${ }^{27}$

No geral, a avaliação dos resultados demonstrou que os laboratórios brasileiros e argentinos foram capazes de determinar a quantidade e a composição isotópica do urânio em níveis esperados em amostras ambientais típicas (da ordem de nanogramas). No

Tabela 4. Resumo das principais informações dos trabalhos citados

\begin{tabular}{|c|c|c|c|c|c|}
\hline Autor & Instituição & Analito & Tipo de Amostra & $\begin{array}{c}\text { Metodologia de Preparação da } \\
\text { Amostra }\end{array}$ & Técnica Analítica \\
\hline Godoy et al. & IRD & $\begin{array}{l}\text { Radioisótopos de } \\
\text { interesse }\end{array}$ & $\begin{array}{c}\text { Água / Solo / } \\
\text { Sedimentos Marítimos }\end{array}$ & Extração (TOPO) e Precipitação & $\begin{array}{c}\text { Espectrometria Alfa } \\
\text { Técnicas de medição de } \\
\text { radioatividade }\end{array}$ \\
\hline Godoy et al. & IRD & Urânio e Plutônio & $\begin{array}{l}\text { Materiais de Referência / } \\
\text { Esfregaço / Solo }\end{array}$ & Extração Cromatográfica & ICP-MS \\
\hline Buchmann & IPEN & Urânio & $\begin{array}{l}\text { Agulhas de Pinheiro } \\
\text { (Bioindicadores) }\end{array}$ & $\begin{array}{l}\text { 1) Lavagem ácida assistida por } \\
\text { ultrassom } \\
\text { 2) Digestão ácida assistida por } \\
\text { micro-ondas } \\
\text { 3) Lixiviação ácida }\end{array}$ & HR-ICP-MS \\
\hline Pestana & IPEN & Urânio & Esfregaço & $\begin{array}{l}\text { 1) Lixiviação assistida por ultras- } \\
\text { som } \\
\text { 2) Digestão total }\end{array}$ & $\begin{array}{c}\text { Medidas de atividade } \alpha \\
\text { MEV-EDS } \\
\text { ICP-MS }\end{array}$ \\
\hline Marin & IPEN & Urânio & Esfregaço & $\begin{array}{c}\text { Análise direta sem preparação da } \\
\text { amostra }\end{array}$ & $\begin{array}{c}\text { Medidas de atividade } \alpha \\
\text { MEV-EDS } \\
\text { LA-IP-MS }\end{array}$ \\
\hline
\end{tabular}


entanto, na maioria dos casos, verifica-se que há contaminação com o urânio do ambiente do laboratório considerando que a concentração de urânio na amostra é muitas vezes menor do que a encontrada no ambiente. ${ }^{27}$

Já os resultados de medições de plutônio na segunda etapa do programa poderiam ser obtidos com maior precisão através da realização de métodos de purificação e separação. No entanto, os resultados obtidos e as incertezas estimadas são suficientes para que conclusões sobre atividades nucleares possam ser obtidas sem que sejam necessários grandes esforços no processamento das amostras. ${ }^{17}$

Todos os laboratórios participantes da segunda etapa do programa da ABACC demonstraram a capacidade de medição da razão Pu-240/ $\mathrm{Pu}-239$ dentro do critério de desempenho apresentado na Tabela 1. A quantidade de $\mathrm{Pu}-242$ presente nessas amostras era muito pequena e, por isso, mesmo os laboratórios de melhor desempenho tiveram dificuldades em medir com precisão este isótopo. Com relação à determinação do plutônio total, nem todos os laboratórios obtiveram a precisão exigida de acordo com a Tabela 1. Isso mostra que é preciso melhorar os procedimentos e a compreensão dos processos envolvidos em alguns dos laboratórios. Uma importante observação dos resultados é a contaminação cruzada com urânio observada em alguns laboratórios devido à natureza de outros trabalhos realizados nesses laboratórios. ${ }^{17}$

Nos trabalhos de Godoy et al. para determinação da quantidade total e de composição isotópica do plutônio e do urânio, é possível verificar uma boa precisão nos resultados quando comparados com as medições realizadas no LLNL. ${ }^{28,29}$

Referente ao trabalho de Buchmann, as grandezas obtidas nas amostras processadas de agulhas de pinheiros foram: a concentração elementar e a razão de abundância isotópica do urânio. As soluções ácidas originadas de amostras coletadas nas proximidades da instalação mencionada apresentaram concentração de $0,1 \mu \mathrm{g} \mathrm{kg}^{-1}$ de urânio e uma razão de abundância isotópica U-235/U-238 de 0,0092 $\pm 0,0002$, enquanto as soluções originadas de amostras coletadas em locais situados há mais de $200 \mathrm{~km}$ de distância desta instalação apresentam um valor de $0,0074 \pm 0,0002 .{ }^{9,31}$

Com relação à comparação dos métodos de processamento das amostras a serem inseridas no instrumento de medição, as seguintes observações foram realizadas: 1) O método de lixiviação ácida produziu soluções nas quais os valores de razão de abundância isotópica de urânio apresentaram as menores incertezas, porém, este método exige maior trabalho e maior tempo de processamento. 2) O método de lavagem ácida assistida por ultrassom resultou em soluções cujos resultados de medição das razões de abundância isotópica do urânio foram semelhantes aos obtidos pelo método de lixiviação ácida, porém é um método mais rápido e requer uma quantidade menor de produtos químicos e vidraria. Sua desvantagem é que apresenta alto risco de contaminação durante o processamento da amostra. 3) O método de digestão ácida assistida por micro-ondas, apesar de apresentar diversas vantagens com relação a metodologia de processamento das amostras (menos mão de obra, menor risco de contaminação, maior rapidez, maior segurança e menor custo) e de fornecer resultados similares aos obtidos através dos outros dois métodos de processamento, não se mostrou adequado para a determinação da razão de abundância isotópica de urânio, uma vez que conduziram a incertezas de medição maiores. ${ }^{9,31}$

O trabalho de Pestana identificou uma composição média do isótopo U-235 variando de $0,01453 \pm 0,00023$ a $0,1824 \pm 0,0015$ e a presença do isótopo U-236. Na comparação entre as metodologias de preparação das amostras, o método de lixiviação assistida por ultrassom se mostrou mais simples, rápido e eficiente quando comparado ao método tradicional de digestão total. ${ }^{6}$
As amostras coletadas e medidas por Marin apresentaram uma composição do isótopo U-235 que variou de 0,023 $\pm 0,007$ a 0,173 $\pm 0,028$. Embora não tenha sido atingida a precisão exigida para medição da composição isotópica apresentada na Tabela 1, a condução desse trabalho demonstrou a aplicabilidade e a agilidade do emprego da técnica LA-ICP-MS para análise de amostras de esfregaço. ${ }^{15} \mathrm{O}$ emprego do sistema de ablação a laser para medir as razões isotópicas do urânio reduziu consideravelmente o tempo de análise das amostras, pois permite realizar as medições diretamente na amostra, eliminando assim as etapas de preparação e digestão dos esfregaços. Além disso, a técnica preserva a amostra (análise não destrutiva), sendo esse um requisito de grande relevância para fins de salvaguardas nucleares. ${ }^{15,32}$

No artigo de comparação do uso das técnicas de ICP-MS e LA-ICP-MS, os autores demonstraram a viabilidade dessas técnicas para análises de urânio em amostras reais de esfregaço. Os resultados obtidos por ambas as técnicas estão em boa concordância e as pequenas diferenças entre os métodos podem ser devido à heterogeneidade das amostras. Porém, devido ao problema conhecido de desnível da ablação a laser, os resultados apresentam maiores incertezas exigindo melhorias para que possam ser úteis e confiáveis para fins de salvaguardas nucleares. ${ }^{30}$

\section{CONCLUSÕES}

Os trabalhos iniciais de Godoy et al. permitiram a determinação de radioisótopos de interesse a partir da mesma alíquota de praticamente qualquer tipo de amostra ambiental (água, solo, sedimentos marítimos). Esses trabalhos deram início ao entendimento de metodologias de preparação de amostras ambientais a serem medidas pela técnica de espectrometria de massas.

Já os trabalhos desenvolvidos por Godoy et al. dentro do programa da ABACC demostraram o potencial e a viabilidade de realização no IRD de medições de amostras ambientais para fins de salvaguardas de acordo com os requisitos estabelecidos para este tipo de medição.

O trabalho de Buchmann demonstrou a potencialidade de emprego do instrumento HR-ICP-MS e da metodologia aplicada na determinação de elementos presentes em baixas quantidades em amostras de origem ambiental. Esse trabalho representa uma significativa contribuição na área de salvaguardas, pois comprovou a viabilidade da utilização de um modelo de controle não intrusivo de monitoração ambiental no fornecimento de indícios e evidências de manipulação de urânio.

O trabalho de Pestana demonstrou a importância da utilização de técnicas analíticas complementares para fornecer maiores informações na interpretação dos resultados obtidos em análises de amostras de esfregaço. Através do seu trabalho, o autor apresentou informações sobre o enriquecimento do urânio e identificação de material reprocessado coerentes com as atividades desenvolvidas na instalação em que as amostras de esfregaço foram coletadas.

$\mathrm{O}$ trabalho de Marin também apresentou resultados consistentes com o material manipulado na instalação. Entretanto, a técnica empregada apresentou uma precisão baixa demonstrando a necessidade de melhoria no procedimento de identificação de partículas individuais para eliminação do problema de heterogeneidade da amostra.

O desenvolvimento do programa da $\mathrm{ABACC}$ com assistência do DOE demonstrou que o Brasil e a Argentina possuem laboratórios com potencial para medir a quantidade e a composição isotópica de urânio e plutônio em níveis esperados em amostras ambientais típicas.

O IRD obteve destaque no seu desempenho nesse programa, visto que as medições da razão isotópica e da concentração de urânio e plutônio em amostras de esfregaço obtiveram níveis de precisão exigidos para esta finalidade. No entanto, muitos dos laboratórios 
brasileiros e argentinos observaram problemas de contaminação e verificaram a necessidade de um ambiente mais controlado para processamento desse tipo de amostras.

A plena capacitação analítica brasileira para medição de amostras ambientais para fins de salvaguardas é de extrema importância para um país que já domina todas as etapas do ciclo do combustível nuclear. O domínio de tal técnica reforçará o comprometimento do Brasil com os acordos internacionais firmados. Além disso, essa capacitação permitirá a confrontação dos resultados obtidos nas análises em amostras coletadas nas inspeções de salvaguardas realizadas nas instalações nucleares brasileiras, o que implica em uma maior segurança para afirmação de suas declarações pacíficas.

Após a realização deste estudo, os autores puderam identificar que as medidas necessárias para a implementação de um programa consistente e confiável de análise de amostras ambientais para fins de salvaguardas nucleares no Brasil são: infraestrutura laboratorial dedicada e adequada, instrumentos analíticos altamente sensíveis e seletivos para análise de elementos a nível de traços, profissionais treinados e especializados, aplicação de conceitos metrológicos para obtenção de resultados confiáveis e sistema de garantia de qualidade implementado e preferencialmente acreditado. Para tanto, é necessário que a instituição na qual este laboratório pertença tenha os recursos necessários para manter todas essas medidas em longo prazo.

\section{REFERÊNCIAS BIBLIOGRÁFICAS}

1. Integrated Support Center for Nuclear Nonproliferation and Nuclear Security - ISCN; Handbook of International Nuclear Safeguards; Ibaraki, Japão, 2016.

2. Fischer, D.; History of the International Atomic Energy Agency - The First Forty Years; Viena, Austria, 1997.

3. Review Conference of the Parties to the Treaty on the Non-Proliferation of Nuclear Weapons, Nova Iorque, Estados Unidos, 2015.

4. International Atomic Energy Agency - IAEA; Delivering Effective Nuclear Verification for World Peace; Viena, Austria, 2016.

5. International Atomic Energy Agency - IAEA; International Safeguards in the Design of Enrichment Plants (IAEA Nuclear Energy Series No. NF-T-4.10); Viena, Austria, 2019.

6. Pestana, R. C. B.; Dissertação de Mestrado, Instituto de Pesquisas Energéticas e Nucleares, Brasil, 2013.

7. Cooley, J. N.; Baute, J. G.; Kalinowski, M. B.; Feichter. J.; Nikkinen, M.; Schlosser, C. Em Verifying Treaty Compliance: Limiting Weapons of Mass Destruction and Monitoring Kyoto Protocol Provisions; Avenhaus, R., Kyriakopoulos, N., Richard, M., Stein, G., eds.; Springer: Berlin, 2006, pp. 61-76, 235-257, 367-387.

8. http://antigo.cnen.gov.br/quem-somos, acessada em outubro de 2021.

9. Buchmann, J. H.; Tese de Doutorado, Instituto de Pesquisas Energéticas e Nucleares, Brasil, 2000.

10. https://www.abacc.org.br/a-abacc/sobre-a-abacc, acessada em outubro de 2021.

11. https://www.gov.br/mme/pt-br/assuntos/noticias/mp-cria-autoridadenacional-de-seguranca-nuclear-ansn, acessada em outubro de 2021.
12. Donohue, D. L.; J. Alloys Compd. 1998, 271-273, 11.

13. Donohue, D. L.; Key Tools for Nuclear Inspections: Advances in Environmental Sampling Strengthen Safeguards; Viena, Austria, 2002.

14. Axelsson, A.; Fischer, D. M; Penkin, M. V.; J. Radioanal. Nucl. Chem. 2009, 282, 725.

15. Marin, R. F.; Dissertação de Mestrado, Instituto de Pesquisas Energéticas e Nucleares, Brasil, 2013.

16. Cooley J.; Environmental Sampling - Technical Workshop on Safeguards, Verification Technologies, and Other Related Experience; Viena, Austria, 1998.

17. Perrotta, J. A.; Hembree, D. M.; Olsen, K.; Russ, P.; Williams, R.; Whitaker, M.; Guidicini, O. M.; Godoy, M. L.; Sarkis, J. E. S.; Kakazu, M.; Pecequilo, B.; SANTOS, A. J. G.; Gautier, E.; Servant, R.; Ledesma, A.; Caravaglia, R.; Esteban, A.; Gómez, J. C.; Bonino, N.; Rosa, S.; Development of Capability to Separate and Measure Uranium and Plutonium Jointly Present in Low-Level Environmental Samples at Brazilian and Argentine Laboratories Working for ABACC.

18. Ticknor, B. W.; Bottorff, S. C.; Hexel, C. R.; Tevepaugh, K. N.; Bostick, D. A.; Automated Clean Chemistry for Bulk Analysis of Environmental Swipe Samples; Oak Ridge, Estados Unidos, 2016.

19. Oliveira Jr., O. P.; Sarkis, J. E. S.; J. Radioanal. Nucl. Chem. 2002, 253, 345.

20. Solatie, D.; Dissertação de Mestrado, Universidade de Helsinque, Finlândia, 2002.

21. Metzger, S. C.; Ticknor, B. W.; Rogers, K. T.; Bostick, D. A.; McBay, E. H; Hexel, C. R.; Anal. Chem. 2018, 90, 9441.

22. International Atomic Energy Agency - IAEA; Safeguards Techniques and Equipment; Viena, Austria, 2011.

23. Shinonaga, T.; Esaka, F.; Magara, M.; Klose, D.; Donohue, D.; Spectrochim. Acta, Part B, 2008, 63, 1324.

24. Donohue, D. L.; Environmental Sample Analysis - Advances and Future Trends; Viena, Austria.

25. Lauria, D. C; Godoy, J. M.; The Science of the Total Environment 1988, $70,83$.

26. Godoy, J. M.; Lauria, D. C.; Godoy, M. L. D. P.; Cunha, R. P.; J. Radioanal. Nucl. Chem. 1994, 182, 165.

27. Guidicini, O. M.; Hembree, D. M. Jr.; Carter, J. A.; Olsen, K.; Hayes, S.; Whitaker, M.; Development of Low-Level Environmental Sampling Capabilities for Uranium at Brazilian and Argentine Laboratories by ABACC.

28. Godoy, M. L. D. P.; Godoy, J. M.; Roldão, L. A.; J. Environ. Radioact. 2007, 97, 124.

29. Godoy, M. L. D. P.; Godoy, J. M.; Roldão, L. A.; Tauhata, L.; J. Environ. Radioact. 2009, 100, 613 .

30. Pestana, R. C. B.; Sarkis, J. E. S.; Marin, R. C.; Abreu-Junior, C. H.; Carvalho, E. F. U.; International Nuclear Atlantic Conference - INAC, Recife, Brasil, 2013.

31. Buchmann, J. H.; Sarkis, J. E.; Kakazu, M. H.; Rodrigues, C.; J. Radioanal. Nucl. Chem. 2006, 270, 291-.

32. Marin, R. C.; Sarkis, J. E. S.; Nascimento, M. R. L.; J. Radioanal. Nucl. Chem. 2013, 295, 99. 